\title{
Energetic, Structural and Interfacial Evolutions in Hydration and Dehydration of Minerals with Layered Structures
}

\author{
Di Wu ${ }^{12}$, HONGWU XU ${ }^{3}$
}

${ }^{1}$ Alexandra Navrotsky Institute for Experimental Thermodynamics, Washington State University, Pullman, Washington 99163, United States

${ }^{2}$ The Gene and Linda Voiland School of Chemical Engineering and Bioengineering, Washington State University, Pullman, Washington 99163, United States ${ }^{3}$ Earth and Environmental Sciences Division, Los Alamos National Laboratory, Los Alamos, New Mexico 87545, United States

Hydration of minerals with layered structures is an important interfacial phenomenon which governs many critical geochemical processes in the Earth's critical zone. Here, I present our recent studies on the energetics, structural and interfacial evolutions during hydration and dehydration of a series of minerals and relevant synthetic layered materials, including clays layered double hydroxides (LDHs), and clay-like transition metal carbides (MXenes). These minerals were studied by both in situ X-ray diffraction (XRD) and in situ diffuse reflectance infrared Fourier transform spectroscopy (DRIFTS) to reveal the structural and interfacial phenomena during dehydration. Moreover, we employed integrated thermogravimetric analysis-differential scanning calorimetry-mass spectrometry (TG-DSC-MS) analyses, solution calorimetry and adsorption calorimetry to elucidate the energetics of water layered mineral interactions. These fundamental studies enhance our fundamental understanding on interfacial chemistry between water and layered minerals on both Earth and near-earth planet such as Mars. 